Sessions d'étude - Société canadienne d'histoire de l'Église catholique

\title{
Le rôle des évêques de Chicoutimi dans l'oeuvre du Séminaire
}

\section{Marius Paré}

Volume 40, 1973

URI : https://id.erudit.org/iderudit/1007250ar

DOI : https://doi.org/10.7202/1007250ar

Aller au sommaire du numéro

Éditeur(s)

Les Éditions Historia Ecclesiæ Catholicæ Canadensis Inc.

ISSN

0318-6172 (imprimé)

1927-7067 (numérique)

Découvrir la revue

Citer cet article

Paré, M. (1973). Le rôle des évêques de Chicoutimi dans l'oeuvre du Séminaire. Sessions d'étude - Société canadienne d'histoire de l'Église catholique, 40,

113-124. https://doi.org/10.7202/1007250ar

Tous droits réservés @ Les Éditions Historia Ecclesiæ Catholicæ Canadensis Inc., 1974
Ce document est protégé par la loi sur le droit d'auteur. L'utilisation des services d’Érudit (y compris la reproduction) est assujettie à sa politique d'utilisation que vous pouvez consulter en ligne.

https://apropos.erudit.org/fr/usagers/politique-dutilisation/ 


\section{Le rôle des évêques de Chicoutimi dans l'œuvre du Séminaire}

Le quarantième congrès annuel de la Société canadienne d'Histoire de l'Église catholique a fait une place généreuse à l'histoire de l'Église dans notre région du Saguenay-Lac-Saint-Jean. Nous sommes sensibles à ce geste de compréhension et d'estime, et nous remercions de tout cœur les dirigeants de la Société qui en sont les auteurs. Notre gratitude ne vient pas de la vanité ou de la candeur mais de notre connaissance de ce que l'Église a accompli dans notre région depuis le début de la colonie française et qui est splendide, de notre volonté aussi de continuer fidèlement cette histoire selon ce que l'Esprit-Saint nous indiquera. Pour vivre et travailler dans la vraie fidélité, il faut se relier à l'ensemble et participer à la vie de l'ensemble. Vous nous en donnez l'occasion et de cela nous vous sommes reconnaissants. Peut-être aussi vous apporterons-nous un concours qui n'est pas sans valeur.

Vous m'avez confié le soin de vous entretenir du rôle des évêques de Chicoutimi dans l'œuvre du Séminaire centenaire. Nous ferons ensemble un survol de cette histoire, car nous ne pouvons faire davantage en un temps restreint. Il nous donnera tout de même l'occasion de constater une fois de plus comment un peuple naît et grandit, comment l'Église naît, croît et établit le règne de Dieu chez ce peuple et lui fait produire une œuvre d'éternité. Ce rappel ne sera pas de trop pour nous aider à vivre aujourd'hui dans la lumière et la vertu.

\section{I \\ L'origine}

Le Séminaire de Chicoutimi a ouvert ses portes le 15 septembre 1873 dans une humble école cédée pour $\$ 1.00$ par MM. les Membres de la Commission scolaire avec la permission du ministère de l'Instruction publique. Son fondateur fut M. l'abbé Dominique Racine, curé de la paroisse SaintFrançois-Xavier depuis 1862. Incontestablement, c'était un homme d'Église et un patriote intelligent, perspicace, expérimenté. Au surplus, un bâtisseur. Dès 1865, il avait eu l'idée d'une école ou d'un collège où l'on donnerait aux jeunes de Chicoutimi et des environs une instruction plus 
poussée et plus appropriée aux besoins de la population de la région que celle reçue dans les écoles primaires.

De 1865 à 1873, l'idée de monsieur Racine évolua. À plusieurs reprises il en causa avec son archevêque, Monseigneur Elzéar-Alexandre Taschereau, et en discuta avec son entourage, si bien qu'au terme c'est un séminaire ou collège classique qu'il fonda à l'instar de plusieurs régions de la province.

\section{II}

\section{Nos collèges classiques}

L'histoire des collèges classiques et le rôle majeur qu'ils ont joué dans la vie de notre peuple et celle de l'Église catholique au Québec est à écrire. Il serait urgent de s'y mettre car nous sommes en train de l'oublier depuis que le ministère de l'Éducation a suscité la disparition de plusieurs d'entre eux et en a réduit d'autres. En dépit des services éminents qu'ils ont rendus à notre communauté française et catholique, ils n'échappent pas actuellement au dédain dont les "nouveaux riches" entourent le passé.

Sous la domination française, nous n'avons eu qu'un seul collège dit classique: le Collège des Jésuites fondé en 1635, auquel se lia en 1668 le Petit Séminaire de Québec. En 1767, sept ans après la conquête, un deuxième s'ajouta: le Collège de Montréal ouvert et soutenu par les Sulpiciens. Au XIX siècle les fondations de séminaires ou collèges classiques se succèdent à un rythme croissant: neuf entre 1800 et 1850 , huit entre 1851 et 1900 . Un peu partout, à mesure que la population augmente et que le pays s'organise, on ressent le mal douloureux qu'est l'ignorance pour un peuple qui veut survivre dans des conditions difficiles en gardant sa foi, sa langue et sa nationalité.

L'Église à défaut de l'État a pris l'initiative. Elle fonda et maintint à coups de sacrifices et de dévouement méconnus ou oubliés ces institutions que, pendant quelque temps, on a appelées nos forteresses et qui ont été parmi les facteurs les plus efficaces de notre survivance ethnique et religieuse. Nous convenons aisément qu'elles aient eu des défauts, mais quand on pense aux conditions de vie qui étaient les leurs et à la pénurie des moyens dont elles disposaient pour atteindre le but fixé, ce qu'elles ont accompli apparaît merveilleux et honore ceux qui les ont soutenues, dirigées et développées.

Pourquoi fondait-on des séminaires-collèges? Mais tout simplement parce qu'ils répondaient aux besoins d'un peuple pour qui vivre et croire se faisaient d'un même mouvement. Les institutions furent à l'image du peuple. La préoccupation de la base et de ses besoins n'est pas tout à fait 
une invention du temps présent. L'on avait besoin de prêtres pour la vie religieuse: il fallait penser séminaire. L'on avait besoin d'hommes instruits, d'hommes de loi, de médecins, d'hommes de lettres et de plus en plus d'hommes de sciences, de professeurs et d'administrateurs pour le service et la gouverne de la cité : il fallait penser collège classique. Et comme tous a vaient la même foi et le même souci de la vivre et que l'on n'avait pas les ressources financières pour multiplier les institutions spécialisées, il ne restait qu'un moyen: le séminaire-collège où chacun trouverait son bien. Institutions qui convenaient à tous mais à personne? Sans minimiser les a vantages de l'organisation actuelle, nous pouvons affirmer que celle d'autrefois avait moins de gros défauts. Depuis 1635 jusqu'au milieu de notre siècle, nos séminaires-collèges nous ont donné les dirigeants religieux et civils dont nous avions besoin et contribuèrent à maintenir chez nous une solidarité ct une harmonie que bien des peuples plus riches et plus puissants pouvaient nous envier.

\section{III}

\section{Monseigneur Dominique Racine et le Séminaire de Chicoutimi}

C'est ce sens de la réalité qui amena Monseigneur Elzéar-Alexandre Taschereau, archevêque de Québec, et monsieur Dominique Racine, son vicaire général au Saguenay depuis mars 1871, à définir l'institution projetée et à fonder un séminaire de préférence à tout autre genre d'institution.

La bénédiction eut lieu le 28 juillet 1873. Elle fut présidée par Monseigneur Taschereau qui était en visite de confirmation au Saguenay. Monseigneur l'Archevêque entourait la nouvelle institution d'une attention constante, généreuse, et soutenait de tout son pouvoir monsieur Racine qui en était le fondateur et le réalisateur. Il lui écrivait, entrant dans les détails de l'organisation, précisant l'esprit qui devra régner dans la communauté et le règlement qu'on devra suivre. Le 15 août suivant, il en fit l'érection canonique et la mit sous le patronage de la Sainte Famille. Tout en maintenant monsieur Racine dans sa charge de curé de la paroisse de Saint-François-Xavier, il le nomma supérieur du nouveau séminaire. Et dès le début, constatant que bientôt la maison serait trop exiguë, il enjoignit le supérieur de voir sans retard à une construction plus conforme aux besoins. Monsieur Racine n'était pas homme à se faire prier quand le devoir lui était présenté avec tant de clarté. Il se mit à la tâche avec décision et courage, prit part à tout, même travailla de ses mains à la construction. De son côté, pour aider à en défrayer le coût, Monseigneur Taschereau et quelques prêtres de Québec firent un don de $\$ 1,000.00$. De plus, l'archevêque demanda à tous les fidèles du diocèse de Québec de 
verser un sou par an pendant trois ans. Si bien que, le 4 août 1875 , il venait encore à Chicoutimi bénir le nouveau séminaire construit en pierre, tout près de l'église paroissiale.

L'attention et la générosité de l'archevêque de Québec à l'endroit du séminaire ne se terminèrent pas là. Des dons réguliers suivirent. Il s'intéressa à la régie interne de la communauté agrandie, à la vie des prêtres et des grands séminaristes tout particulièrement. Cet intérêt persistant s'explique en ce qu'il songeait à faire de la région du Saguenay-LacSaint-Jean une circonscription ecclésiastique autonome. Il en fait état dans son décret canonique du 15 août 1873, en énumérant les motifs de la fondation: "... l'importance toujours croissante du district du Saguenay, la difficulté extrême qu'il a de communiquer avec le centre du diocèse pendant une grande partie de l'année, et la pensée que cette région pourra, dans un avenir plus ou moins éloigné, former un diocèse distinct."

Le 28 mai 1878, c'était chose faite. Monsieur Dominique Racine devenait le premier évêque de Chicoutimi. Il fut sacré à Québec le 4 août et intronisé le 7 août suivant par son métropolitain et ami, Monseigneur Elzéar-Alexandre Taschereau.

Devenu évêque et chef du diocèse, Monseigneur Racine demeura supérieur du séminaire jusqu'au 14 juin 1882 . Il se fit même procureur de 1876 à 1881 et professeur de théologie pour les grands séminaristes. À la demande de son archevêque, il était venu résider au nouveau séminaire afin de mieux suivre sa vie et son développement. Il ne le quitta qu'en 1887 , quelques mois avant sa mort qui survint en janvier 1888. Non seulement nous devons considérer Monseigneur Racine comme l'initiateur, le fondateur et le réalisateur de cette œuvre, mais comme celui qui en fut l'intelligence et le cœur pendant les quinze premières années de son existence.

“C'est par ses soins, écrit Monseigneur André Simard dans son livre: Les évêques et les prêtres séculiers du diocèse de Chicoutimi, que cette institution obtint son affiliation à l'Université Laval de Québec le 22 mai 1877 et fut érigée en corporation civile par acte du Parlement provincial le 31 octobre 1879, que la Congrégation de la Sainte Vierge fut établie le 25 décembre 1880 et que saint Thomas d'Aquin lui fut donné comme patron des études le 25 février 1885. Il rédigea lui-même les Constitutions concernant le gouvernement et l'administration de la maison, Constitutions qui furent mises en vigueur le 21 janvier 1882 et qu'il complétera dans la suite par ses "Notes sur les devoirs des régents ou maîtres de discipline du Petit Séminaire de Chicoutimi" livrées le 21 décembre 1883 et par ses "Notes sur les devoirs des professeurs " livrées le 30 décembre suivant. " "Oeuvre d'amour ", écrira-t-il, au sujet des Constitutions. 
Au surplus, il donna pour le soutien matériel de son séminaire tout ce qu'il lui était possible de donner, même la dîme qu'il recevait comme curé de la cathédrale. À la fin, pour couronner le tout, il lui laissa son cœur qui a été conservé dans la chapelle et qui attend actuellement la nouvelle localisation du séminaire pour y être transporté.

À MM. les abbés Ambroise Fafard, curé d'office, et Thomas Roberge, secrétaire à l'évêché, qui le veillaient dans la nuit qui précéda sa mort, il dit : “Mon séminaire... Mon séminaire, comme je l'aimais! Je puis dire de lui comme Adam de la compagne que Dieu lui avait donnée: "C'était l'os de mes os, la chair de ma chair! " J'aurais voulu faire pour lui beaucoup plus que je n'ai fait: Dieu ne l'a pas permis ${ }^{1}$. "Et pourtant, que pouvait-il faire de plus?

\section{Les successeurs}

Monseigneur Dominique Racine avait fondé et organisé l'œuvre primordiale du séminaire diocésain. Il lui avait donné son premier élan. Depuis 1880, chaque année, des finissants quittaient soit pour le Grand Séminaire ou les communautés religieuses, soit pour l'Université. Il avait ordonné son premier prêtre, ancien du séminaire, pour le diocèse en 1883. Il en ordonnera 26 autres. En mourant en 1888, il laissait à ses successeurs une œuvre inestimable dont bénéficiaient déjà largement l'Église et la société au Saguenay et au Lac-Saint-Jean. Tous lui gardent un attachement et une reconnaissance qui vont jusqu'à la vénération.

Monseigneur Louis-Nazaire Bégin, le futur cardinal-archevêque de Québec, fut le premier de ses successeurs. Il dirigea le diocèse d'octobre 1888 à 1892, d'abord comme évêque résidentiel, puis comme administrateur pendant la vacance du siège qui suivit sa nomination à Québec comme coadjuteur de Son Éminence le Cardinal Taschereau.

Ancien professeur au Petit puis au Grand Séminaire de Québec, ancien principal de l'École normale Laval de Québec il ne pouvait, par ce qu'il avait été dans le passé, qu'avoir une estime particulière pour le séminaire du diocèse que le Saint-Père lui confiait. Comme son prédécesseur, quoique dans des circonstances différentes, il en fit son "œuvre de prédilection".

En moins de quatre ans, il réussit à la consolider et à la développer. D'abord, il en prit lui-même la direction comme supérieur, ce qui est tout

1 Monseigneur Victor-A. Huard, dans $L$ 'Apôtre du Saguenay. p. 85. 
de même un geste significatif, et se maintint au poste jusqu'à son départ pour Québec. Il se fit professeur de théologie chez les grands séminaristes, encouragea les études supérieures chez les professeurs, envoya à Rome, de ses propres deniers, deux d'entre eux, jeunes prêtres, qui rendront plus lard au séminaire et au diocèse des services remarquables comme professeurs, supérieurs et hommes d'œuvres: MM. les abbés JosephAlfred Tremblay et Elzéar Delamarre. Quelques mois seulement après son arrivée, il fit commencer la construction d'une aile, car, avec l'augmentation du nombre des élèves et des professeurs, l'on était à l'étroit. Le projet a vait été formé et préparé par Monseigneur Racine, mais celui-ci n'avait pu le mettre à exécution. Cette aile nouvelle fut un bienfait. Elle devint le bâtiment principal de l'ensemble et doubla sa capacité. Monseigneur Bègin profita de la circonstance pour compléter, rafraîchir et réaménager l'intérieur du premier corps de bâtiment.

Le 20 avril 1890, il publia un mandement pour établir une quête annuelle en faveur du séminaire. "Nous sommes profondément convaincu, écrivait-il, comme nous l'avons toujours été, que ce séminaire est le plus puissant moyen, non seulement de faire fleurir les fortes études dans le clergé, mais encore de former la jeunesse à la science et aux vertus chrétiennes, de préparer de bons citoyens à la patrie et surtout de recruter de vaillants soldats pour la sainte milice de Jésus-Christ, des prêtres exemplaires pour le ministère des âmes."

En même temps, préoccupé de souder ses efforts à ceux de Monseigneur Racine, il rendait à ce dernier un hommage qui n'avait rien d'équi voque. "Les sacrifices énormes que (Monseigneur Racine) s'est imposés pour donner une base solide à cette institution naissante, Dieu seul les connaît; il la savait essentielle pour la formation et le recrutement de son clergé, en même temps que pour la haute éducation intellectuelle de la jeunesse de son diocèse; il a dépensé toutes les ressources de son énergie et n'a reculé devant aucun sacrifice, devant aucune privation pour assurer l'existence de cette grande œuvre sur laquelle il a déversé toute l'affection dont était capable son âme ardente et généreuse."

Monseigneur Michel-Thomas Labrecque nous venait aussi de Québec où il a vait été professeur et directeur du Grand Séminaire. "Je serai jaloux de l'honneur et de la dignité de mon clergé", a-t-il écrit dans son mandement d'entrée au diocèse, le 22 mai 1892. Un pareil idéal le tournait déjà vers le séminaire où se préparaient les futurs prêtres. Aussi le manifestet-il sans délai par des décisions qui étaient de nature à favoriser le progrès de l'institution. 
Comme son prédécesseur, il se réserva les fonctions de supérieur et les remplit jusqu'en mai 1896. Comme lui aussi, il assuma l'enseignement d'une partie de la théologie, ce qui l'intégrait tout à fait à la vie de la communauté et lui permettait de lui rendre de nombreux services.

Le séminaire avait à ce moment une dette de $\$ 25,000.00$, somme considérable pour cette époque; elle entravait sa marche et son rendement. Le $1^{\text {er }}$ mai 1893, Monseigneur Labrecque adressa une circulaire à ses prêtres et leur demanda de verser pendant 20 ans $2 \%$ de leur revenu au séminaire, afin de lui permettre de boucler. « Pour vous encourager à faire généreusement le léger sacrifice demandé aujourd'hui, écrivait-il, rappelez-vous que l'œu vre du Séminaire diocésain est tellement importante que, sans elle, toutes les autres œuvres religieuses seraient bientôt frappées de langueur et de dépérissement."

Ce secours permit, en effet, de rétablir les finances et même, quelques années après, de construire une chapelle. Mais dans la vie d'un séminaire rien n'est jamais terminé. Il doit évoluer et se développer selon les exigences de la vie en société et tendre à la stature qui convient. Il devenait de constatation quotidienne que la maison ne suffisait plus. L'on était dans la situation ou de marquer le pas, même péricliter, ou agrandir. Monseigneur Labrecque et les directeurs firent le second choix. L'évêque s'adressa cette fois à tous ses diocésains qui étaient immédiatement intéressés à ce que l'institution de haut savoir du diocèse se tienne à jour puisqu'ils lui confiaient leurs enfants. Le 15 août 1907, il écrivit à son peuple pour lui demander de verser cinq centins par an pendant cinq ans au lieu d'un centin comme l'avait établi Monseigneur Bégin.

En 1912, l'on ouvrit le chantier. Tout allait normalement lorsque, dans l'avant-midi du 24 juin, le feu se déclare à l'hôtel Château-Saguenay et, poussé par un fort vent d'ouest, tourne en conflagration. Cent quatre maisons de la ville, le couvent du Bon-Pasteur, la cathédrale et le séminaire sont rasés. Un vrai désastre. Évidemment, l'évêque n'était plus seul pour porter une telle épreuve, mais sa responsabilité l'obligeait à prendre la part la plus considérable: celle de l'étude, de la décision, du courage et de l'aide financière. Monseigneur Labrecque fut à la hauteur. En 1913, la nouvelle construction débuta à l'endroit que l'on connaît et, en septembre 1914, les élèves étaient reçus dans un séminaire tout neuf, mais bien différent du précédent. À cela, cependant, il y avait un envers que nous pressentons: celui des finances. L'évêque jugea qu'il lui appartenait d'intervenir. Il le fit le 26 juillet suivant par une circulaire adressée à son clergé lui demandant de recueillir au cours de la visite paroissiale les charités que la générosité des fidèles leur inspirera. Il y reviendra en 1918 et sollicitera des diocésains qu'ils doublent leur con- 
tribution annuelle de cinq centins. Il interviendra encore en 1921. Luimême donne l'exemple. D'une demande à une autre, d'une souscription à une autre, il a trouvé personnellement quelque $\$ 70,000.00$.

Son attention se tourne encore vers les jeunes bien doués dont les familles sont dans l'incapacité de payer entièrement les études. Il encourage et appuie fortement M. l'abbé Elzéar Delamarre dans la fondation de l'Oeuvre du Petit Séminariste dont le but est de venir en aide aux étudiants qui sont dans cette situation. Au jour de ses cinquante ans de sacerdoce, il fait un don de $\$ 10,000.00$ au Séminaire; l'intérêt sera annuellement attribué à des étudiants dans le besoin. Que ceux qui écrivent et disent que le système d'autrefois n'était ouvert qu'aux riches se donnent la peine de fouiller les archives de nos séminaires. "Drôle de riches!" disait récemment Monseigneur Ovide-Dolor Simard.

Pour un, Monseigneur Labrecque était lié à son séminaire. Pour un, le séminaire de Chicoutimi était lié au peuple qu'il desservait.

En 1921, Monseigneur Labrecque écrivit au supérieur du temps, Monseigneur Eugène Lapointe, une lettre pour appuyer la campagne de souscription que le séminaire organisait pour la construction de l'aile des classes. "Parmi tant d'admirables institutions d'enseignement ou de charité déjà établies dans mon diocèse, écrivait-il, il n'en est aucune, vous le savez, dont j'aie tant à cœur le bon fonctionnement et la prospérité à tout point de vue que le Séminaire, tant à cause de son exceptionnelle importance pour la bonne formation des clercs et d'une élite de laïques, qu'à cause des innombrables bienfaits de tout ordre, spirituels et temporels, qui, par cela même, en jaillissent comme de leur source pour se répandre en flots abondants sur tous les fidèles confiés à ma sollicitude pastorale.

"Aussi me suis-je appliqué avec le plus grand soin dès le début de mon épiscopat, et incessamment jusqu'à ce jour, selon les inclinations de mon cœur et conformément au devoir de ma charge, non seulement à vous aider à y faire fleurir la piété et les bonnes mœurs et à y maintenir à un degré élevé le niveau des études, mais aussi à contribuer dans la mesure de mes moyens à assurer la stabilité de sa situation financière, de laquelle, il n'y a pas à se le dissimuler, dépend en bonne partie tout le reste." La pensée et le cœur de Monseigneur Labrecque sont là. Ils l'ont constamment inspiré au cours des 35 années qu'il dirigea le diocèse de Chicoutimi.

Nous sommes maintenant en 1928. Monsieur l'abbé Charles Lamarche, curé de la paroisse de Saint-Stanislas à Montréal, nommé évêque de Chicoutimi le 17 août 1928, prend possession de son siège le 17 octobre suivant. 
Le séminaire, après les années d'épreuves et de réorganisation que nous avons esquissées, a pris vraiment son essor. Le rôle de l'évêque durant ces années en est un de haute direction, de soutien et d'encouragement. Ce rôle, Monseigneur Lamarche, par sa culture, son doigté, sa bonté et son zèle éclairé le remplit à la perfection dans les circonstances où il se trou vait. Ce qui ne l'empêcha pas d'apporter à l'occasion le secours matériel toujours nécessaire. Il demande aux prêtres de continuer à verser comme par le passé $5 \%$ de leur revenu pour l'œuvre du séminaire. Luimême ne voulut pas s'en dispenser. En 1938 il lui faisait avec la discrétion qui le caractérisait en tout, mais davantage encore en ce qui regardait la charité, un don de $\$ 5,000.00$.

Il ressort de cette histoire que nos séminaires ont été constamment soutenus par le clergé et par le peuple. Cette histoire, elle n'est pas banale. Elle est pleine d'instruction pour qui croit à la vertu pour édifier et former des hommes, voire un peuple. La vertu, elle était présente et elle faisait des merveilles.

Monseigneur Charles Lamarche décède en 1940. Monseigneur Georges Melançon lui succède. C'est pendant la guerre. Une grande activité règne dans la région et durera quelque vingt ans, ce qui impose au pasteur du diocèse un rythme de vie accéléré et une vigilance continuelle. Le séminaire se ressent de ces conditions générales d'existence et de vie. II réclame sa part d'attention. Monseigneur Melançon ne recule pas devant les mesures à prendre. Il a le coup d'œil et la décision rapides. En 1941, il demande à ses diocésains de verser annuellement au séminaire $\$ 2.00$ par famille pour solder les dettes et préparer une construction qui doit contenir la chapelle et l'auditorium.

Dans un mandement substantiel daté du $1^{\text {er }}$ novembre 1941 , il écrit aux fidèles ces lignes qui donnent sa pensée sur le séminaire: "Toutes les œuvres, on en conviendra facilement, n'ont pas une égale importance. Il en est qui pourvoient à des besoins particuliers, qui sont au bénéfice d'une portion restreinte de la population, ou encore qui sont avant tout d'ordre temporel. D'autres sauvegardent les intérêts essentiels de la masse. Telles sont en général les œuvres d'éducation; telle est en particulier l'œuvre du séminaire.

..."Sauver un séminaire, disait saint Jean-Eudes, «c'est sauver les sauveurs, diriger les directeurs, éclairer ceux qui sont la lumière du monde, sanctifier ceux qui sont la sanctification de l'Église". De lui et de lui seul sortira le clergé diocésain, ceux qui seront les pasteurs de vos âmes, à vous tous et chacun de vous, Nos très chers frères. Les autres 
œuvres peuvent être excellentes, infiniment utiles; celle du séminaire est nécessaire, primordiale. Les intérêts éternels, les seuls qui comptent en définitive, sont ici en jeu. C'est l'œuvre mère, celle de laquelle dépendent toutes les autres."

La mesure prise par Monseigneur Melançon donna au séminaire la possibilité d'acquitter la dette, de recueillir des fonds pour la construction de la chapelle et de l'auditorium, projet que l'on réalisa en 1952.

Entre-temps, le séminaire met à contribution ses anciens, célèbre brillamment son $75^{\mathrm{e}}$ anniversaire et complète l'aile de l'ouest, habitée dans sa partie supérieure par les grands séminaristes. En 1950, Monseigneur Melançon prit une décision importante qui transforma l'institution telle qu'elle avait existé jusqu'à ce temps. Depuis 1873, en effet, le grand séminaire et le petit séminaire ne formaient qu'une institution sous la même autorité et la même administration. En cette année 1950, l'évêque déclara le Grand Séminaire autonome et lui donna des constitutions particulières, décision qui lui valut le titre de second fondateur du Grand Séminaire. Une souscription générale diocésaine faite en 1957 permit de construire le grand séminaire où il est actuellement.

Les grand séminaristes habitent cette maison depuis septembre 1958.

\section{V \\ Regard sur le présent et sur l'avenir}

Et voilà ce que les évêques de Chicoutimi ont accompli pour le séminaire centenaire de notre diocèse et pour l'Église. Qu'en pense l'évêque actuel?

Aux fêtes du $75^{\mathrm{e}}$ anniversaire, Monseigneur Melançon, répondant à l'adresse que monsieur le supérieur Joseph Dufour lui présentait, disait : "En feuilletant les mandements, les lettres pastorales, les discours des Évêques de Chicoutimi, on constate qu'il n'y a pas d'œuvre, depuis 75 ans, sur laquelle ils ont insisté davantage, et sur laquelle ils sont revenus plus souvent, pour dire et redire combien ils l'estimaient; pour demander aux fidèles d'en comprendre l'impérieuse nécessité et pour solliciter l'aide généreuse qui lui a permis de grandir et de prospérer."

L'évêque actuel ne croit pas devoir penser ni faire autrement ni plus ni moins. D'abord parce qu'il est envoyé par l'Église pour faire œuvre d'Église. Il doit vivre et agir en Église. Or le Concile de Trente demande aux évêques d'établir un séminaire dans leur diocèse pour préparer les futurs prêtres. Le deuxième concile du Vatican qui parle ou écrit pour nous qui vivons dans la deuxième partie du $\mathrm{XX}^{\mathbf{e}}$ siècle, dans son décret $O p$. tatam totius, numéros 5 et 7 , n'y change rien. Tout en admettant qu'il doit 
être adapté aux nouvelles conditions de vie, il regarde comme acquise l'existence d'un séminaire diocésain ou régional. La Sacrée Congrégation pour l'Éducation Catholique, chargée de l'application du décret conciliaire, insiste pour qu'il en soit ainsi malgré les idées qui circulent. L'Église prend donc clairement position. Elle sait ce qu'elle veut. De plus, ce que nous constatons nous-mêmes et qui est notre expérience personnelle est éclairant. S'il reste aux catholiques que nous sommes un peu d'instinct de conservation, nous maintiendrons nos séminaires dans les meilleures conditions possibles de perfection. C'est ce que nous entendons faire.

En 1963, pour répondre à de nouvelles exigences de l'Université et du Gouvernement du Québec, avec l'appui financier et le contrôle de ce dernier, le séminaire a commencé la construction de bâtiments considérables comprenant des laboratoires, des salles de cours, des bureaux, un auditorium, un gymnase, une piscine et deux grandes résidences. Il occupa les nouveaux locaux deux ans après. L'amélioration était incontestable mais il a vait contracté une dette de deux millions de dollars.

En 1964, apparut le ministère de l'Éducation qui en peu de temps renouvela entièrement notre système d'éducation au Québec de façon à coordonner tout l'enseignement et à le rendre accessible à tous. Entre autres, il institua le Collège d'enseignement général et professionnel qui amputa notre cours classique de ses trois dernières années. Nos professeurs et nos élèves passèrent à ce nouveau Collège.

Le Gouvernement offrit au séminaire d'acheter ses bâtiments et le terrain avoisinant. Pour plusieurs raisons le séminaire décida de collaborer et d'accepter la vente. Les tractations furent longues, mais on finit par s'entendre. Au terme, le séminaire, réduit aux classes du secondaire et à une résidence au plan collégial, doit se loger ailleurs. Nous espérons que ce sera fait à l'automne 1974. Nous voulons qu'il vive, avant tout pour les raisons exposées ci-haut. Que sera-t-il au juste? Une institution privèe à but vocationnel, reconnue officiellement d'intérêt public par le ministère de l'Éducation. Il se limite actuellement aux classes du secondaire mais il entrevoit la possibilité de donner des cours au niveau collégial selon qu'il sera utile ou nécessaire à ses élèves. Il ne veut pas entrer en compétition avec les autres institutions. Il veut simplement poursuivre sa fin qui est de donner à l'Église les hommes dont elle a besoin, sachant que, par le fait même, il rend à la société un service éminent. Celle-ci n'a pas de meilleure collaboratrice et servante que l'Église. Il est ouvert, selon notre tradition à nous, à tous ceux qui acceptent sa foi, son esprit et son régime de vie. 
Chers congressistes, le Séminaire de Chicoutimi qui vient de célébrer son centenaire s'est identifié avec la région. Vous ne pouvez circuler dans nos villes et paroisses sans rencontrer des hommes qui sont venus chercher en ses murs les connaissances intellectuelles et professionnelles, les convictions morales et religieuses, l'idéal et le style de vie qui leur ont permis de donner bonne mesure dans la vie et d'aider notre peuple à devenir ce qu'il est et qui n'est pas médiocre. Nous passons, il est vrai, par la tourmente comme les autres peuples. Nous éprouvons des difficultés. Nous voulons en sortir heureusement, sans déchéance. C'est à quoi le Séminaire de Chicoutimi travaille et veut travailler comme il l'a fait depuis cent ans. "Admire la cime, pense aux racines " a été le mot d'ordre inspiré par un jeune étudiant du secondaire IV pour l'année du centenaire. Les racines qui plongent dans le sol vivent dans l'obscurité, mais la santé de l'arbre, le prestige de la cime, la beauté du feuillage et la qualité des fruits dépendent de la qualité des racines qui vont chercher dans les profondeurs les aliments nécessaires à tout l'arbre. Le séminaire, ce sont les racines. Pour atteindre la cime, nous pensons aux racines.

† Marius PARÉ,
Évêque de Chicoutimi.

\section{Sources}

- Archives de l'évêché de Chicoutimi: dossiers des évêques du diocèse et du Séminaire de ('hicoutimi.

- Mandements, Lettres pastorales et Circulaires des Évêques de Chicoutimi, publiés à partir de 1878.

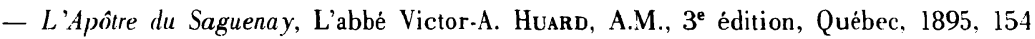
pages.

- Monseigneur Dominique Racine - Bâtisseur de l'Église saguenéenne, Jean-Claude Drolet. Publications de La Société Historique du Saguenay. n 20. Chicoutimi, 1968, XIX-236 pages.

- Les Evếques et les Prêtres séculiers au Diocèse de Chicoutimi 1878-1968, Notices biographiques, (M ${ }^{\mathrm{gr}}$ ) André Simard, Chicoutimi, 1969, 813 pages.

- Saguenayensia, revue de La Société Historique du Saguenay, livraison de septembreoctobre 1972 .

- Une Gerbe de Souvenirs 1873-1973 ou le Séminaire raconté par ses Anciens, Chicoutimi. 1973, 211 pages.

- Mémoire de la Fédération des Collèges classiques à la Commission royale d'enquête sur less problèmes constitutionnels, Montréal, Fides, 1954.

- Décret Optatam totius du Deuxième Concile du Vatican. 